\title{
Fine-scale genetic analyses reveal unexpected spatial-temporal heterogeneity in two natural populations of the commercial mushroom Agaricus bisporus
}

\author{
Jianping $\mathrm{Xu}_{1}{ }^{1}$ Christophe Desmerger ${ }^{2}$ and Philippe Callac ${ }^{3}$
}

\begin{abstract}
Author for correspondence: Jianping Xu. Tel: +1 9055259140 ext. 27934. Fax: +1 9055226066. e-mail: jpxu@mcmaster.ca
\end{abstract}

1 Department of Biology, McMaster University, 1280 Main Street West, Hamilton, Ontario, Canada L8S 4K1

2 Centre Technique du Champignon, Munet, 49400 Distré, France

3 Institute National de la Recherche Agronomique, Unité de Recherche sur les Champignons, B.P. 81 , 33883 Villenave d'Ornon, France

\begin{abstract}
This study examined the fine-scale genetic variation of the commercial mushroom, Agaricus bisporus, over 2 years at two sites in France. One site was a meadow fertilized with horse manure and disturbed regularly by humans; the other was a Monterey cypress forest free of human disturbance. Altogether, $\mathbf{5 0}$ mushrooms were collected and analysed for mitochondrial and nuclear genetic variation marked by RFLPs and multilocus enzyme electrophoretic polymorphisms. Population samples from these two sites were genetically different and both sites contained high levels of genetic diversity. No identical genotypes were found at either site between the 2 years and there was little evidence for extensive vegetative clonality for this species. Contrary to expectations, very limited evidence of pseudohomothallic reproduction was found. Results from tests of Hardy-Weinberg equilibrium and genotypic equilibrium showed that outcrossing and recombination have played significant roles in both populations. The results demonstrated spatialtemporal genetic heterogeneity of $\boldsymbol{A}$. bisporus in natural populations.
\end{abstract}

Keywords: molecular markers, modes of reproduction, population structure, fungi

\section{INTRODUCTION}

The application of molecular markers has had significant impact in addressing questions concerning the natural history and population biology of many organisms, clarifying modes of reproduction, speciation and the spatial-temporal dynamics of populations. For the commercial button mushroom, Agaricus bisporus Lange (Imbach) (syn. A. brunnescens Peck), large-scale geographic structure has been investigated, primarily due to the potential utility of wild germplasm for commercial breeding purposes (Callac, 1995; Kerrigan, 1995; Xu et al., 1997, 1998). However, relatively little is known about the mode of reproduction and the fine-scale spatial-temporal dynamics of A. bisporus in natural environments. The purpose of this study was to use highly polymorphic molecular markers to investigate the potential modes of reproduction and patterns of spatial-temporal genetic variation of A. bisporus in two defined locations in France.

Abbreviations: GE, genotypic equilibrium; HWE, Hardy-Weinberg equilibrium; LG, linkage group.
The basidiomycete $A$. bisporus is a major agricultural crop in many countries, with a worldwide annual production worth over US\$2 billion (Chang, 1993). This fungus has been cultivated for about three centuries, beginning in France during the reign of Louis XIV. The practice subsequently spread to other European countries. By the early twentieth century, commercial cultivation of A. bisporus was widespread on all populated continents. Recent genetic evidence indicated that escapes of cultivar germplasm to the wild accompanied the spread of commercial cultivation in many geographic areas (Xu et al., 1997, 1998).

The mating system in A. bisporus is controlled by one locus with multiple alleles (Xu et al., 1993, 1996a; Imbernon et al., 1995). Homokaryons (i.e. mycelium with only one nuclear type) with different alleles at the mating-type locus can mate to form a heterokaryon (i.e. mycelium with two different nuclear types) capable of forming mushroom fruiting bodies. However, the production of homokaryotic basidiospores is limited in most strains of A. bisporus (Allen et al., 1992; Kerrigan et al., 1993; Royse \& May, 1982; Summerbell et al., 1989). Most often, the majority of basidia produce only 
two basidiospores with each receiving two of the four post-meiotic nuclei. These two nuclei are often nonsisters with respect to meiosis II and carry compatible mating types. Basidiospores from these two-spored basidia germinate to produce fertile heterokaryotic mycelia in which the two genetically different nuclei coexist in each hyphal compartment. This life cycle is termed pseudohomothallism or secondary homothallism (Raper et al., 1972; Royse \& May, 1982). Pseudohomothallic reproduction in A. bisporus tends to maintain the parental genotype in the offspring (Allen et al., 1992; Kerrigan et al., 1993; Summerbell et al., 1989).

In addition to pseudohomothallic life cycle, heterothallism (outcrossing) can also occurs in A. bisporus. Some basidia produce three or four basidiospores, many of which receive only one post-meiotic nucleus. Such basidiospores can germinate to form self-sterile homokaryons. Mating among homokaryons with compatible mating types from the same or different mushroom fruiting bodies can result in the formation of a fertile heterokaryon (Xu et al., 1993, 1996a). Therefore, through this life cycle, inbreeding (or selfing) and outcrossing are possible in A. bisporus. Because of the two modes of reproduction, pseudohomothallism and heterothallism, A. bisporus was also termed an amphithallic species (Lange, 1952). The alternative life cycles of A. bisporus depend on the ploidy level of the spores, a trait correlated to the number of spores per basidium.

Despite extensive laboratory studies, few data are available on the modes of reproduction in natural populations of A. bisporus. An earlier report based on samples from different areas in California suggested evidence of pseudohomothallic reproduction in the Californian populations of A. bisporus (Kerrigan et al., 1998). Those inferences were tentative, not supported by direct observation of mushroom from the same site over time (Kerrigan et al., 1998). Two factors contribute to this lack of understanding. The first is the lack of appropriate samples for such an analysis. Unlike many basidiomycete species, no method is currently available for the direct isolation of $A$. bisporus mycelia from substrates in nature. Collections are limited only to strains obtained from mushroom fruiting bodies either directly from nature, or, rarely, indirectly by placing natural inoculum (e.g. leaf litter or horse manure) onto fruiting substrates under optimal fruiting conditions in the lab and isolating mushrooms from these fruiting experiments (Callac et al., 2000). Unfortunately, fruiting of A. bisporus is influenced by many factors (e.g. temperature, moisture and substrate) and is not a commonly observed event in nature.

The second factor contributing to our lack of understanding of reproductive systems in nature is the difficulty in distinguishing outcrossing from inbreeding and pseudohomothallic reproduction. All three processes can generate novel genotypes. However, with highly polymorphic genetic markers, specific predictions from these reproductive modes could be directly compared. Specifically, outcrossing generates random as- sociation of alleles at the same or different locus and the observed and expected heterozygosities should be comparable. By contrast, long-term strict inbreeding and pseudohomothallic reproduction could not generate random associations of alleles within and between loci. With strict inbreeding, the overall observed heterozygosity should be lower than those expected under random mating. By contrast, on a short time scale, pseudohomothallic reproduction in A. bisporus could maintain original parental genotype and heterozygosity, but the maintenance of such heterozygosity is not expected on a long-term scale.

The objective of this study was to examine the mode of reproduction and the fine-scale population structure of A. bisporus. Samples from two sites in France were monitored and examined over a 2-year period. One site contained horse manure as substrate and had frequent human disturbances (site 1). The other site was associated with Monterey cypress trees and was without human disturbance (site 2). Highly polymorphic genetic markers were used to examine the genetic structure within and between these two sites.

\section{METHODS}

Sites and strains. Favourable mushroom fruiting conditions existed in two sites in France over a 2-year period. These two sites were located about $450 \mathrm{~km}$ apart. Site 1 was in Gradignan, near Bordeaux, and covered an area of about $50 \times 70 \mathrm{~m}$. Site 2 was in Dinard, near Saint Malo, and covered an area of about $20 \times 30 \mathrm{~m}$. The nearest known sample of $A$. bisporus was at least $3 \mathrm{~km}$ from site 1 and about $300 \mathrm{~m}$ from site 2 . The habitat in site 1 was a meadow where horse manure has been spread each May since 1985. Site 2 is near the English Channel in a Monterey cypress (Cupressus macrocarpa) forest. The trees were planted in 1947 and the site lacked human disturbance. The numbers of isolates found in each site and year are presented in Table 1. Isolates were identified as A. bisporus based on their morphological, and in some cases biochemical, features and on the ability of homokaryons from these heterokaryons to form heterokaryons when paired with known A. bisporus homokaryons. Sites 1 and 2 were previously reported as sites 13 and 24, respectively, with respect to the collecting of A. bisporus in France (Callac, 1994; Guinberteau et al., 1998). At both sites during the first year, only one representative from each cluster of fruiting bodies was isolated and analysed. Different fruiting bodies from the same cluster (i.e. lumped at the base) were usually identified as genetically identical and likely they resulted from the same vegetative mycelia (see Results). However, to obtain a more complete sample, all the fruiting bodies for the second year at both sites were collected and analysed. In total, 50 fruiting bodies were isolated. No fruiting body from the second-year sample was found at exactly the same location as ones from the previous year at either site. Because of the lack of correlation between physical distances among strains and their genetic distances within each sample (see Results), physical locations of mushroom fruiting bodies are not presented. For genotype analysis, except for strain BS78L where multi-spore culture was used, all other strains were derived directly from fruiting body tissues. The mean spore number per basidium was determined for each of the 50 strains (Callac, 1994; Guinberteau et al., 1998). As far as we know, this collection of 50 fruiting bodies represents the best 
material available for fine-scale spatial-temporal analysis of genetic variation of $A$. bisporus from natural environments.

Marker assay. Three types of co-dominant molecular markers were assayed for all 50 strains. The first type was based on Southern hybridization of cloned single-copy DNA fragments to EcoRI-digested total genomic DNA. This method screened RFLPs for 10 nuclear loci and 9 mitochondrial probes. The 10 nuclear loci were 1n17, 1n36, 1n38, 1n55, 1n148, 4n6, 4n27, 33n5, 33n16 and 33n18 (Xu et al., 1997; Kerrigan et al., 1993, 1996; Table 1). The 9 mitochondrial probes were B4b, B5, B6, B7, B9, B13, B15, BS1 and BS2(5) (Hintz et al., 1988; Jin \& Horgen, 1993; Xu et al., 1998). Allelic determinations and nomenclature followed those of a global population genetic survey by Xu et al. (1997).

The second genotyping method was based on PCR of singlecopy DNA fragments, followed by restriction digests of the PCR products to detect RFLPs (PCR-RFLP). Five polymorphic PCR-RFLP markers were assayed (PR2, PR3b, PR4, PR5 and PR6). The third type of molecular marker is based on allozyme polymorphism at three loci, alcohol dehydrogenase (ADH), phosphoglucomutase (PGM), and $\beta$-glucosidase (BGLU). Locus name and allelic designations for each strain are presented in Table 1. Detailed information for individual loci can be found elsewhere (Callac et al., 1997; Hintz et al., 1988; Jin \& Horgen, 1993; Kerrigan et al., 1993, 1996; Mouyna \& Brygoo, 1993; Xu et al., 1997, 1998) and are available upon request from J.Xu (jpxu@mcmaster.ca) and P. Callac (callac@ bordeaux.inra.fr). Mitochondrial DNA (mtDNA) haplotypes in Table 1 were determined based on the composite Southern hybridization patterns of nine mtDNA probes to EcoRIdigested total genomic DNA as described by Xu et al. (1998). These nine probes covered about $60 \%$ of the mtDNA in a cultivated strain (Hintz et al., 1988).

Data analysis. Each mushroom fruiting body was considered an independent isolate. Multilocus genotypes were identified for each of the 50 strains. Standard population genetic measures of mean alleles per locus, observed heterozygosity $(\mathrm{Ho})$ and expected heterozygosity (= gene diversity, $\mathrm{He}$ ) were calculated. The distribution of genetic diversity within and among samples and the degree of correlation among uniting gametes were evaluated through $F$-statistics. $F$-statistics were calculated by using the ANOVA approach presented by Weir \& Cockerham (1984). Inbreeding coefficients were calculated as recommended by Reynolds et al. (1983). The program Genetic Data Analysis GDA1d16c (version 1.0D16c) by P. O. Lewis and D. Zaykin was used for the above calculations of population genetic parameters. This program was kindly provided by Paul Lewis of the University of Connecticut, USA.

To determine if there was evidence for outcrossing in natural populations of $A$. bisporus, we performed two tests. The first compared the observed genotype frequencies with those expected under the assumption of random mating (test of Hardy-Weinberg equilibrium, HWE). The lack of significant difference between observed and expected genotypic counts would be consistent with HWE and the hypothesis that outcrossing and recombination have played a significant role in these populations. The second test was to determine the associations of genotypes between loci [test of genotypic equilibrium (GE)] for each sample. For the GE tests, diploid genotypes with each locus were preserved to ensure that disequilibrium within a locus (i.e. the Hardy-Weinberg disequilibrium) does not influence the tests between loci.
Because of multiple tests, Bonferoni correction was used to adjust significance values for the tests of genotypic disequilibrium (Zaykin et al., 1995). In this test, random associations of genotypes between loci would also be consistent with recombination and suggest evidence of outcrossing. Fisher's exact tests were used to determine both HWE and GE for each sample. Statistical significances of both tests were based on the observed genotypic frequency and compared to the null distribution of genotype frequencies. The null genotype frequency distributions were derived from permutation with 1000 repeats using empirical allele frequencies and under the null hypothesis of random mating. Through permutation, the random effects of small sample sizes on both HWE and GE are reduced. Tests were done for each individual locus and between all pairs of loci. The program GDA1d16c was used for these two disequilibrium tests (Lewis \& Zaykin, 2001).

While outcrossing and recombination were tested against the null hypotheses of random mating, the alternative hypotheses of clonality, inbreeding and pseudohomothallic reproduction were tested directly through pairwise comparisons of multilocus genotypes. To determine whether inbreeding and pseudohomothallic reproduction are present and if so, their prevalence, we compared all pairs of strains between the two years from each site and looked for evidence of highly similar or identical genotypes. Specifically, identical genotypes and loss of heterozygosity shown in the second-year sample would suggest such reproductions in nature. Beyond mutation, no gain of heterozygosity should be expected for pseudohomothallic reproduction or inbreeding. In addition, vegetative clonality could also contribute to identical genotypes between the years. However, loss of heterozygosities for nuclear loci was only consistent with inbreeding or pseudohomothallic reproduction.

\section{RESULTS}

\section{Molecular genetic variation}

The genotypes of all strains are presented in Table 1 . There were high levels of genetic variation in all four samples (Table 2). At site 1 , all 18 nuclear loci in the 1991 sample were polymorphic, while 16 were polymorphic in the 1992 sample. At site 2, all 18 loci were polymorphic for both the 1992 and 1993 samples. The mean number of alleles among the 18 nuclear loci was $2 \cdot 9$ (site 1, 1991), $2 \cdot 7$ (site 1, 1992), $3 \cdot 5$ (site 2, 1992) and $2 \cdot 9$ (site 2, 1993) (Table 2).

From Southern hybridization of nine mtDNA probes, a total of nine mtDNA haplotypes (A, B, C, D, E, F, G, H and I) were found among the 50 strains analysed in this study. Briefly, there were two mtDNA haplotypes (A and C) in the site 1, 1991, sample; four haplotypes (A, C, $\mathrm{D}$ and $\mathrm{E})$ in the site 1, 1992, sample; seven haplotypes (A, B, C, D, F, G and H) in the site 2, 1992, sample; and five haplotypes (A, B, G, H and I) in the site 2, 1993, sample. mtDNA haplotype A was present in all four samples. This haplotype is one of the two mtDNA haplotypes in the cultivar strains used for at least the last two decades worldwide for commercial cultivation (Kerrigan, 1995; Xu et al., 1998). Haplotype B is the other cultivar mtDNA haplotype. This haplotype was found in both samples from site 2, but not site 1 (Table 1). 
Table 1. Multilocus genotypes of $50 \mathrm{~A}$. bisporus strains analysed in this study

See Methods for references to loci information.

\begin{tabular}{|c|c|c|c|c|c|c|c|c|c|c|c|c|c|c|c|c|c|c|c|}
\hline \multirow[t]{2}{*}{ Strain } & \multicolumn{18}{|c|}{ Nuclear* } & \multirow{2}{*}{$\begin{array}{l}\text { mtDNA* } \\
\text { haplotype }\end{array}$} \\
\hline & $\ln 148$ & PR5 & PR2 & PR6 & $\ln 17$ & $4 n 6$ & $33 n 5$ & $33 \mathrm{n} 16$ & $33 n 18$ & $\ln 38$ & $\ln 55$ & $\ln 36$ & $4 n 27$ & Adh & Pgm & Glu & PR3b & PR4 & \\
\hline \multicolumn{20}{|c|}{ Site 1, Gradignan, 1991 sample } \\
\hline BS78A & $3 / 6$ & $3 / 3$ & $2 / 2$ & $1 / 3$ & $2 / 4$ & $2 / 3$ & $8 / 8$ & $6 / 6$ & $3 / 3$ & $1 / 7$ & $6 / 6$ & $4 / 4$ & $1 / 2$ & $3 / 3$ & $2 / 2$ & $6 / 6$ & $1 / 1$ & $2 / 2$ & $\mathrm{~A}$ \\
\hline BS78B & $3 / 6$ & $1 / 5$ & $1 / 2$ & $1 / 1$ & $2 / 4$ & $2 / 4$ & $3 / 8$ & $6 / 6$ & $3 / 3$ & $1 / 7$ & $6 / 8$ & $4 / 4$ & $2 / 3$ & $3 / 5$ & $2 / 5$ & $5 / 5$ & $1 / 2$ & $1 / 2$ & $\mathrm{~A}$ \\
\hline BS78C & $3 / 6$ & $3 / 4$ & $2 / 2$ & $1 / 1$ & $4 / 4$ & $3 / 3$ & $8 / 8$ & $6 / 6$ & $3 / 3$ & $1 / 7$ & $6 / 8$ & $3 / 6$ & $1 / 2$ & $4 / 5$ & $2 / 5$ & $6 / 6$ & $1 / 2$ & $2 / 3$ & $\mathrm{~A}$ \\
\hline BS78D & $4 / 6$ & $5 / 7$ & $1 / 2$ & $1 / 1$ & $2 / 4$ & $2 / 3$ & $3 / 3$ & $6 / 7$ & $3 / 4$ & $1 / 7$ & $6 / 8$ & $5 / 5$ & $1 / 2$ & $3 / 5$ & $2 / 5$ & $6 / 6$ & $2 / 2$ & $1 / 2$ & $\mathrm{~A}$ \\
\hline BS78E & $3 / 6$ & $1 / 5$ & $1 / 2$ & $1 / 1$ & $4 / 4$ & $3 / 3$ & $4 / 8$ & $4 / 7$ & $3 / 3$ & $1 / 1$ & $8 / 8$ & $5 / 5$ & $1 / 2$ & $3 / 7$ & $5 / 5$ & $6 / 6$ & $1 / 2$ & $2 / 2$ & C \\
\hline BS78F & $3 / 4$ & $5 / 7$ & $1 / 2$ & $1 / 4$ & $2 / 4$ & $3 / 4$ & $5 / 8$ & $6 / 6$ & $3 / 3$ & $1 / 7$ & $8 / 8$ & $4 / 4$ & $1 / 2$ & $2 / 5$ & $2 / 5$ & $5 / 5$ & $2 / 2$ & $1 / 2$ & $\mathrm{C}$ \\
\hline BS78G & $3 / 6$ & $3 / 3$ & $2 / 2$ & $1 / 3$ & $2 / 4$ & $2 / 4$ & $8 / 8$ & $6 / 6$ & $3 / 4$ & $1 / 7$ & $6 / 8$ & $4 / 4$ & $2 / 3$ & $3 / 5$ & $2 / 5$ & $5 / 5$ & $1 / 1$ & $2 / 2$ & $\mathrm{~A}$ \\
\hline BS78H & $3 / 6$ & $1 / 5$ & $1 / 2$ & $1 / 1$ & $4 / 4$ & $3 / 3$ & $4 / 8$ & $6 / 6$ & $3 / 3$ & $1 / 1$ & $8 / 8$ & $4 / 4$ & $1 / 2$ & $3 / 7$ & $5 / 5$ & $6 / 6$ & $1 / 2$ & $2 / 2$ & $\mathrm{C}$ \\
\hline BS78I & $3 / 6$ & $1 / 5$ & $1 / 2$ & $1 / 1$ & $2 / 4$ & $2 / 3$ & $8 / 8$ & $6 / 6$ & $3 / 3$ & $1 / 7$ & $6 / 6$ & $4 / 4$ & $1 / 2$ & $3 / 3$ & $2 / 2$ & $6 / 6$ & $1 / 2$ & $1 / 1$ & $\mathrm{~A}$ \\
\hline BS78J & $3 / 6$ & $1 / 5$ & $2 / 2$ & $1 / 3$ & $2 / 4$ & $3 / 4$ & $8 / 8$ & $6 / 6$ & $3 / 3$ & $1 / 7$ & $6 / 8$ & $4 / 6$ & $1 / 2$ & $3 / 5$ & $2 / 5$ & $6 / 6$ & $2 / 2$ & $1 / 2$ & $\mathrm{~A}$ \\
\hline BS78K & $3 / 4$ & $3 / 3$ & $1 / 2$ & $1 / 4$ & $2 / 2$ & $3 / 4$ & $5 / 5$ & $6 / 6$ & $3 / 4$ & $1 / 7$ & $6 / 8$ & $4 / 7$ & $1 / 2$ & $3 / 4$ & $2 / 5$ & $6 / 6$ & $1 / 1$ & $2 / 2$ & A \\
\hline \multicolumn{20}{|c|}{ Site 1, Gradignan, 1992 sample } \\
\hline BS78L & $3 / 6$ & $3 / 3$ & $2 / 2$ & $1 / 3$ & $4 / 4$ & $3 / 3$ & $5 / 8$ & $6 / 6$ & $3 / 3$ & $1 / 1$ & $8 / 8$ & $4 / 4$ & $1 / 2$ & $3 / 7$ & $5 / 5$ & $6 / 6$ & $1 / 1$ & $2 / 2$ & $\mathrm{C}$ \\
\hline BS78M & $3 / 6$ & $3 / 3$ & $2 / 2$ & $1 / 3$ & $4 / 4$ & $3 / 3$ & $5 / 8$ & $6 / 6$ & $3 / 3$ & $1 / 1$ & $8 / 8$ & $4 / 4$ & $1 / 2$ & $3 / 3$ & $5 / 5$ & $6 / 6$ & $1 / 1$ & $2 / 2$ & $\mathrm{C}$ \\
\hline BS78N & $3 / 6$ & $3 / 3$ & $2 / 2$ & $1 / 3$ & $4 / 4$ & $3 / 3$ & $5 / 8$ & $6 / 6$ & $3 / 3$ & $1 / 1$ & $8 / 8$ & $4 / 4$ & $1 / 2$ & $3 / 3$ & $5 / 5$ & $6 / 6$ & $1 / 1$ & $2 / 2$ & C \\
\hline BS78P & $4 / 6$ & $5 / 7$ & $1 / 2$ & $1 / 1$ & $2 / 4$ & $2 / 3$ & $4 / 4$ & $6 / 6$ & $3 / 4$ & $1 / 7$ & $5 / 8$ & $4 / 4$ & $2 / 2$ & $5 / 7$ & $2 / 2$ & $6 / 6$ & $1 / 2$ & $2 / 2$ & $\mathrm{~A}$ \\
\hline BS78Q & $4 / 6$ & $5 / 7$ & $1 / 2$ & $1 / 1$ & $2 / 4$ & $2 / 3$ & $4 / 4$ & $6 / 6$ & $3 / 4$ & $1 / 7$ & $5 / 8$ & $4 / 4$ & $2 / 2$ & $5 / 7$ & $2 / 2$ & $6 / 6$ & $1 / 2$ & $2 / 2$ & A \\
\hline BS78R & $4 / 6$ & $5 / 7$ & $1 / 2$ & $1 / 1$ & $2 / 4$ & $2 / 3$ & $4 / 4$ & $6 / 6$ & $3 / 4$ & $1 / 7$ & $5 / 8$ & $4 / 4$ & $2 / 2$ & $5 / 7$ & $2 / 2$ & $6 / 6$ & $1 / 2$ & $2 / 2$ & $\mathrm{~A}$ \\
\hline BS78T & $3 / 6$ & $3 / 4$ & $2 / 2$ & $1 / 1$ & $3 / 3$ & $3 / 4$ & $4 / 6$ & $6 / 6$ & $3 / 3$ & $1 / 6$ & $6 / 6$ & $6 / 6$ & $1 / 2$ & $3 / 5$ & $2 / 5$ & $6 / 6$ & $1 / 2$ & $1 / 1$ & $\mathrm{D}$ \\
\hline BS78U & $3 / 6$ & $3 / 4$ & $2 / 2$ & $1 / 1$ & $3 / 3$ & $3 / 4$ & $4 / 6$ & $6 / 6$ & $3 / 3$ & $1 / 6$ & $6 / 6$ & $6 / 6$ & $1 / 2$ & $3 / 5$ & $2 / 5$ & $6 / 6$ & $1 / 2$ & $1 / 1$ & $\mathrm{D}$ \\
\hline BS78V & $3 / 6$ & $3 / 4$ & $2 / 2$ & $1 / 1$ & $3 / 3$ & $3 / 4$ & $4 / 4$ & $6 / 6$ & $3 / 3$ & $1 / 6$ & $6 / 6$ & $6 / 6$ & $1 / 2$ & $3 / 5$ & $2 / 5$ & $6 / 6$ & $1 / 2$ & $1 / 1$ & $\mathrm{D}$ \\
\hline BS78Y & $3 / 6$ & $1 / 5$ & $1 / 2$ & $1 / 1$ & $3 / 3$ & $2 / 3$ & $3 / 8$ & $6 / 6$ & $3 / 3$ & $3 / 5$ & $6 / 6$ & $4 / 7$ & $1 / 2$ & $3 / 3$ & $2 / 2$ & $6 / 6$ & $1 / 1$ & $1 / 2$ & $\mathrm{~A}$ \\
\hline BS78Z & $3 / 6$ & $3 / 3$ & $2 / 2$ & $1 / 3$ & $4 / 4$ & $3 / 3$ & $5 / 8$ & $6 / 6$ & $3 / 3$ & $1 / 1$ & $8 / 8$ & $4 / 4$ & $1 / 2$ & $3 / 3$ & $5 / 5$ & $6 / 6$ & $1 / 1$ & $2 / 2$ & $\mathrm{E}$ \\
\hline \multicolumn{20}{|c|}{ Site 2, Dinard, 1992} \\
\hline BS257 & $3 / 6$ & $3 / 3$ & $2 / 2$ & $1 / 4$ & $4 / 4$ & $3 / 4$ & $4 / 8$ & $6 / 6$ & $3 / 3$ & $1 / 5$ & $5 / 8$ & $4 / 4$ & $1 / 3$ & $2 / 5$ & $4 / 5$ & $6 / 6$ & $1 / 2$ & $1 / 2$ & $\mathrm{~A}$ \\
\hline BS258 & $3 / 4$ & $5 / 7$ & $1 / 2$ & $1 / 4$ & $2 / 4$ & $3 / 4$ & $3 / 3$ & $6 / 6$ & $3 / 4$ & $1 / 7$ & $6 / 8$ & $4 / 6$ & $2 / 5$ & $5 / 7$ & $2 / 5$ & $5 / 5$ & $1 / 2$ & $2 / 2$ & $\mathrm{~B}$ \\
\hline BS259 & $3 / 3$ & $1 / 6$ & $2 / 2$ & $3 / 4$ & $2 / 4$ & $3 / 4$ & $4 / 4$ & $3 / 6$ & $3 / 4$ & $7 / 9$ & $6 / 8$ & $4 / 6$ & $1 / 2$ & $3 / 3$ & $2 / 5$ & $2 / 6$ & $1 / 2$ & $2 / 2$ & $\mathrm{~A}$ \\
\hline BS260 & $3 / 6$ & $3 / 3$ & $2 / 2$ & $1 / 4$ & $4 / 4$ & $3 / 4$ & $4 / 4$ & $6 / 6$ & $3 / 3$ & $1 / 5$ & $5 / 8$ & $4 / 4$ & $1 / 3$ & $2 / 5$ & $4 / 5$ & $6 / 6$ & $1 / 2$ & $1 / 2$ & G \\
\hline BS261 & $3 / 3$ & $5 / 7$ & $1 / 2$ & $1 / 4$ & $2 / 4$ & $2 / 6$ & $4 / 8$ & $6 / 6$ & $3 / 3$ & $1 / 3$ & $8 / 8$ & $5 / 9$ & $1 / 5$ & $3 / 3$ & $3 / 5$ & $2 / 2$ & $1 / 2$ & $2 / 2$ & A \\
\hline BS262 & $3 / 4$ & $5 / 7$ & $1 / 2$ & $1 / 4$ & $2 / 4$ & $3 / 4$ & $4 / 4$ & $6 / 6$ & $3 / 4$ & $7 / 9$ & $8 / 8$ & $4 / 4$ & $2 / 5$ & $3 / 3$ & $2 / 3$ & $6 / 6$ & $2 / 2$ & $1 / 2$ & $\mathrm{H}$ \\
\hline BS263 & $3 / 3$ & $1 / 6$ & $2 / 2$ & $3 / 4$ & $2 / 4$ & $3 / 4$ & $4 / 4$ & $3 / 6$ & $3 / 4$ & $7 / 9$ & $6 / 8$ & $4 / 6$ & $1 / 2$ & $3 / 3$ & $2 / 2$ & $3 / 3$ & $1 / 2$ & $2 / 2$ & A \\
\hline BS264 & $3 / 6$ & $1 / 5$ & $2 / 2$ & $1 / 3$ & $2 / 4$ & $3 / 4$ & $6 / 6$ & $6 / 6$ & $3 / 3$ & $1 / 7$ & $6 / 8$ & $4 / 6$ & $1 / 2$ & $3 / 5$ & $2 / 5$ & $6 / 6$ & $2 / 2$ & $1 / 2$ & $\mathrm{~A}$ \\
\hline BS265 & $3 / 6$ & $1 / 5$ & $2 / 2$ & $1 / 3$ & $2 / 4$ & $3 / 4$ & $6 / 6$ & $6 / 6$ & $3 / 3$ & $1 / 7$ & $6 / 8$ & $4 / 6$ & $1 / 2$ & $3 / 5$ & $2 / 5$ & $6 / 6$ & $2 / 2$ & $1 / 2$ & A \\
\hline BS266 & $3 / 6$ & $1 / 5$ & $2 / 2$ & $1 / 3$ & $2 / 4$ & $3 / 4$ & $6 / 6$ & $6 / 6$ & $3 / 3$ & $1 / 7$ & $6 / 8$ & $4 / 6$ & $1 / 2$ & $3 / 5$ & $2 / 5$ & $6 / 6$ & $2 / 2$ & $1 / 2$ & A \\
\hline BS267 & $3 / 4$ & $5 / 7$ & $1 / 2$ & $1 / 4$ & $2 / 4$ & $3 / 4$ & $4 / 4$ & $6 / 6$ & $3 / 4$ & $1 / 7$ & $8 / 8$ & $4 / 4$ & $2 / 5$ & $3 / 3$ & $2 / 2$ & $6 / 6$ & $2 / 2$ & $1 / 2$ & $\mathrm{C}$ \\
\hline BS268 & $3 / 4$ & $5 / 7$ & $1 / 2$ & $1 / 4$ & $2 / 4$ & $3 / 4$ & $4 / 4$ & $6 / 6$ & $3 / 4$ & $1 / 7$ & $8 / 8$ & $4 / 4$ & $2 / 5$ & $3 / 3$ & $2 / 3$ & $6 / 6$ & $2 / 2$ & $1 / 2$ & C \\
\hline BS269 & $3 / 4$ & $5 / 7$ & $1 / 2$ & $1 / 4$ & $2 / 4$ & $3 / 4$ & $4 / 4$ & $6 / 7$ & $3 / 4$ & $1 / 7$ & $8 / 8$ & $4 / 4$ & $2 / 5$ & $3 / 3$ & $2 / 2$ & $6 / 6$ & $2 / 2$ & $1 / 2$ & C \\
\hline BS270 & $3 / 6$ & $3 / 3$ & $2 / 2$ & $1 / 4$ & $4 / 4$ & $3 / 4$ & $4 / 4$ & $6 / 6$ & $3 / 3$ & $1 / 5$ & $5 / 8$ & $4 / 4$ & $1 / 3$ & $2 / 5$ & $4 / 5$ & $6 / 6$ & $1 / 2$ & $1 / 2$ & A \\
\hline BS271 & $3 / 4$ & $5 / 7$ & $1 / 2$ & $1 / 4$ & $2 / 4$ & $3 / 3$ & $5 / 5$ & $4 / 6$ & $3 / 3$ & $1 / 6$ & $5 / 8$ & $4 / 6$ & $1 / 5$ & $7 / 7$ & $2 / 2$ & $3 / 3$ & $1 / 2$ & $1 / 1$ & $\mathrm{D}$ \\
\hline BS272 & $3 / 4$ & $5 / 7$ & $1 / 2$ & $1 / 4$ & $2 / 4$ & $3 / 4$ & $4 / 4$ & $6 / 7$ & $3 / 4$ & $7 / 7$ & $8 / 8$ & $4 / 4$ & $1 / 5$ & $3 / 3$ & $2 / 2$ & $6 / 6$ & $2 / 2$ & $1 / 2$ & $\mathrm{~F}$ \\
\hline BS274 & $3 / 4$ & $5 / 7$ & $1 / 2$ & $1 / 4$ & $2 / 4$ & $4 / 4$ & $3 / 3$ & $6 / 6$ & $3 / 4$ & $1 / 7$ & $6 / 8$ & $4 / 6$ & $2 / 5$ & $5 / 7$ & $2 / 5$ & $5 / 5$ & $1 / 1$ & $2 / 2$ & B \\
\hline \multicolumn{20}{|c|}{ Site 2, Dinard, 1993} \\
\hline BS355 & $3 / 4$ & $5 / 7$ & $1 / 2$ & $1 / 4$ & $2 / 4$ & $4 / 4$ & $3 / 3$ & $6 / 6$ & $3 / 4$ & $1 / 7$ & $6 / 8$ & $4 / 6$ & $2 / 5$ & $5 / 7$ & $2 / 5$ & $5 / 5$ & $1 / 2$ & $2 / 2$ & $\mathrm{~B}$ \\
\hline BS357 & $3 / 4$ & $1 / 5$ & $1 / 2$ & $1 / 1$ & $2 / 4$ & $2 / 4$ & $8 / 8$ & $6 / 6$ & $3 / 4$ & $1 / 7$ & $6 / 8$ & $4 / 4$ & $2 / 3$ & $3 / 5$ & $2 / 5$ & $5 / 5$ & $1 / 2$ & $1 / 1$ & $\mathrm{~A}$ \\
\hline BS358 & $3 / 4$ & $5 / 7$ & $1 / 2$ & $1 / 4$ & $2 / 4$ & $3 / 4$ & $4 / 4$ & $6 / 6$ & $3 / 4$ & $1 / 7$ & $8 / 8$ & $4 / 4$ & $2 / 5$ & $3 / 3$ & $2 / 2$ & $6 / 6$ & $2 / 2$ & $2 / 2$ & I \\
\hline BS359 & $3 / 4$ & $5 / 7$ & $1 / 2$ & $1 / 4$ & $2 / 4$ & $3 / 4$ & $4 / 4$ & $6 / 6$ & $3 / 4$ & $1 / 7$ & $8 / 8$ & $4 / 4$ & $2 / 5$ & $3 / 3$ & $2 / 2$ & $6 / 6$ & $2 / 2$ & $1 / 2$ & I \\
\hline BS361 & $3 / 4$ & $5 / 7$ & $1 / 2$ & $1 / 4$ & $2 / 4$ & $3 / 4$ & $4 / 4$ & $6 / 6$ & $3 / 4$ & $1 / 7$ & $8 / 8$ & $4 / 4$ & $2 / 5$ & $3 / 3$ & $2 / 5$ & $6 / 6$ & $2 / 2$ & $1 / 2$ & $\mathrm{H}$ \\
\hline BS363 & $3 / 3$ & $1 / 5$ & $1 / 2$ & $1 / 4$ & $2 / 4$ & $3 / 3$ & $4 / 6$ & $6 / 6$ & $3 / 4$ & $1 / 1$ & $8 / 8$ & $4 / 4$ & $1 / 1$ & $3 / 3$ & $5 / 5$ & $6 / 6$ & $2 / 2$ & $2 / 2$ & I \\
\hline BS364 & $3 / 6$ & $5 / 7$ & $2 / 2$ & $1 / 4$ & $4 / 4$ & $3 / 4$ & $4 / 8$ & $6 / 6$ & $3 / 4$ & $1 / 7$ & $8 / 8$ & $6 / 6$ & $1 / 2$ & $5 / 5$ & $2 / 5$ & $6 / 6$ & $2 / 2$ & $1 / 1$ & G \\
\hline
\end{tabular}


Table 1 (cont.)

\begin{tabular}{|c|c|c|c|c|c|c|c|c|c|c|c|c|c|c|c|c|c|c|c|}
\hline \multirow[t]{2}{*}{ Strain } & \multicolumn{18}{|c|}{ Nuclear* } & \multirow{2}{*}{$\begin{array}{l}\text { mtDNA* } \\
\text { haplotype }\end{array}$} \\
\hline & $\ln 148$ & PR5 & PR2 & PR6 & $\ln 17$ & $4 n 6$ & $33 n 5$ & $33 \mathrm{n} 16$ & $33 \mathrm{n} 18$ & $\ln 38$ & $\ln 55$ & $\ln 36$ & $4 n 27$ & Adh & Pgm & Glu & PR3b & PR4 & \\
\hline BS365 & $4 / 6$ & $5 / 7$ & $1 / 2$ & $1 / 4$ & $2 / 4$ & $3 / 4$ & $4 / 4$ & $6 / 6$ & $3 / 4$ & $1 / 5$ & $8 / 8$ & $4 / 4$ & $2 / 5$ & $3 / 3$ & $2 / 2$ & $6 / 6$ & $2 / 2$ & $1 / 2$ & $\mathrm{H}$ \\
\hline BS368 & $3 / 3$ & $5 / 7$ & $1 / 2$ & $1 / 4$ & $2 / 3$ & $3 / 4$ & $4 / 5$ & $6 / 6$ & $? / ?$ & $1 / 7$ & $5 / 8$ & $4 / 6$ & $2 / 2$ & $3 / 3$ & $32 / 5$ & $6 / 6$ & $2 / 2$ & $3 / 3$ & $\mathrm{H}$ \\
\hline BS369 & $3 / 4$ & $5 / 7$ & $1 / 2$ & $1 / 4$ & $2 / 4$ & $3 / 4$ & $4 / 5$ & $6 / 6$ & $3 / 4$ & $1 / 7$ & $5 / 8$ & $4 / 6$ & $1 / 2$ & $3 / 3$ & $2 / 5$ & $6 / 6$ & $2 / 2$ & $2 / 2$ & $\mathrm{H}$ \\
\hline BS370 & $3 / 3$ & $1 / 6$ & $2 / 2$ & $3 / 4$ & $2 / 4$ & $3 / 4$ & $4 / 4$ & $3 / 6$ & $3 / 4$ & $7 / 9$ & $6 / 8$ & $4 / 6$ & $1 / 2$ & $3 / 3$ & $32 / 2$ & $6 / 6$ & $1 / 2$ & $2 / 2$ & A \\
\hline
\end{tabular}

*For descriptions of types of markers, see Methods. Allelic nomenclatures of the nuclear and mitochondial RFLP loci followed those established by Xu et al. (1997) during a global survey of genetic variation for A. bisporus. These data are available upon request from J. Xu(jpxu@mcmaster.ca). Primer information and nomenclature of alleles for PCR-RFLP and allozyme loci are available from P. Callac (callac@bordeaux.inra.fr). The loci are arranged according to their linkage relationships. Loci 1n148, PR5, PR2, PR6 and 1n147 are on linkage group (LG) I; locus 4n6 on LG II; loci 33n5 and 33n16 on LG III, locus 33n18 on LG V; locus 1n38 on LG VI; and locus 1 n36 on LG X. Linkage relationships for the remaining 7 loci (4n27, Est1, Adh, Pgm, Glu, PR3b and PR4) are not known at present.

\section{Genotype distributions and vegetative clonality}

Vegetative clonality in A. bisporus is inferred from multilocus genotypic analysis of fruiting bodies. Based on the combined nuclear and mitochondrial genotypic data, no evidence of vegetative clonality was found in two of the four samples: the site 1,1991, sample and the site 2, 1993, sample. All fruiting bodies from each of these two samples were genetically unique (Table 1). In the site 1, 1992, sample, three genotypes were shared among seven strains. However, each shared genotype was from mushrooms clustered together with almost no physical distance from each other. The first cluster contained isolates BS78M, BS78N and BS78Z. The second contained isolates BS78P, BS78Q and BS78R. The third contained BS78T, BS78U and BS78V. Therefore, isolates from site 1, 1992, did not provide any robust evidence for extensive vegetative clonality of A. bisporus (Table 1). In fact, physically clustered fruiting bodies can have different genotypes either in the mitochondrial genome (BS78M and BS78N versus $\mathrm{BS78Z}$ ) or in the nuclear genome (BS78T and BS78U versus BS78V) (Table 1). The total diameters of the basal areas for each of the three clusters in the site 1 , 1992, sample did not exceed $10 \mathrm{~cm}$.
The only evidence of potential vegetative clonality came from the site 2, 1992, sample. The three isolates BS264, BS265 and BS266 were found to have identical multilocus genotypes (Table 1). BS264 was $0.4 \mathrm{~m}$ from BS265; BS265 about $1.0 \mathrm{~m}$ from BS266; and BS264 about $1.3 \mathrm{~m}$ from BS266. Aside from these clusters of mushrooms, there was no correlation between genetic distance and physical distance among the strains at either site (data not shown).

\section{Difference between the two sites}

Statistical analysis confirmed that populations from sites 1 and 2 were significantly differentiated from each other (Table 3). The overall $F_{\mathrm{ST}}$ value (inbreeding coefficient between sub-populations) was 0.0727 with $95 \%$ confidence interval of $0 \cdot 0402-0 \cdot 1061$, not overlapping with 0 . Within each site, the differences between years were statistically not significant; the $95 \%$ confidence intervals all overlapped 0 (Table 3 ). At site 2, both samples had higher observed heterozygosity than those expected under random mating (Table 3 ). However, the two samples from site 1 differed in patterns of heterozygosity, with the 1991 sample having higher observed

Table 2. Descriptive population genetic statistics for samples of $A$. bisporus

\begin{tabular}{|c|c|c|c|c|c|c|}
\hline Site & Year & $\boldsymbol{P}^{*}$ & $A \dagger$ & $\mathrm{He} \ddagger$ & $H o \mathbb{S}$ & $\mathrm{IC} \|$ \\
\hline \multirow[t]{2}{*}{ 1. Gradignan } & 1991 & $1 \cdot 0$ & $2 \cdot 9$ & $0 \cdot 52$ & $0 \cdot 56$ & -0.079 \\
\hline & 1992 & $0 \cdot 89$ & $2 \cdot 7$ & $0 \cdot 46$ & $0 \cdot 41$ & $0 \cdot 102$ \\
\hline \multirow[t]{2}{*}{ 2. Dinard } & 1992 & $1 \cdot 0$ & $3 \cdot 5$ & $0 \cdot 55$ & $0 \cdot 63$ & $-0 \cdot 151$ \\
\hline & 1993 & $1 \cdot 0$ & $2 \cdot 9$ & $0 \cdot 49$ & $0 \cdot 58$ & $-0 \cdot 209$ \\
\hline
\end{tabular}

$* P$, proportion of polymorphic loci (maximum value is $1 \cdot 0$ ).

$\dagger A$, mean number of alleles per locus.

$\ddagger$ He, mean expected heterozygosity per locus (= gene diversity).

$\$ H o$, mean observed heterozygosity per locus.

\| IC, inbreeding coefficient estimated based on the method-of-moments (Reynolds et al., 1993). 
Table 3. Similarity and difference among samples of $A$. bisporus in two French sites

Mean and $95 \%$ confidence interval (CI) for each estimate are presented. $F_{\mathrm{IS}}, F_{\mathrm{IT}}, F_{\mathrm{ST}}$, inbreeding coefficients within a sub-population, within the total population and between sub-populations, respectively; NS, not statistically significant.

\begin{tabular}{|c|c|c|c|}
\hline & $F_{\mathrm{IS}}$ & $F_{\mathrm{IT}}$ & $F_{\mathrm{ST}}$ \\
\hline \multicolumn{4}{|c|}{ Site 1 , between years 1 and 2} \\
\hline Mean & $0 \cdot 0067$ & $0 \cdot 0316$ & $0 \cdot 0251$ (NS) \\
\hline $95 \% \mathrm{CI}$ & $-0 \cdot 1566$ to $0 \cdot 1784$ & $-0 \cdot 1411$ to $0 \cdot 2123$ & -0.0083 to 0.0621 \\
\hline \multicolumn{4}{|c|}{ Site 2 , between years 1 and 2} \\
\hline Mean & $-0 \cdot 1710$ & $-0 \cdot 1714$ & $-0.0004(\mathrm{NS})$ \\
\hline $95 \%$ CI & $-0 \cdot 3430$ to $0 \cdot 0366$ & $-0 \cdot 3392$ to $0 \cdot 0289$ & $-0 \cdot 0123$ to $0 \cdot 0151$ \\
\hline \multicolumn{4}{|c|}{ Between sites 1 and 2} \\
\hline Mean & $-0 \cdot 0857$ & $0 \cdot 0042$ & $0 \cdot 0727 *$ \\
\hline $95 \% \mathrm{CI}$ & $-0 \cdot 2499$ to $0 \cdot 0910$ & $-0 \cdot 1449$ to $0 \cdot 1631$ & $0 \cdot 0402$ to $0 \cdot 1061$ \\
\hline
\end{tabular}

$* P<0 \cdot 05$, significant difference between sites 1 and 2 .

heterozygosity than expected and the 1992 sample showing the reverse (Table 2).

\section{Lack of identical genotypes between the two years at either site}

We were interested in whether mushrooms with the same multilocus genotypes could be recovered repeatedly at a site over a 2-year period. If so, then there was potential evidence for vegetative mycelial longevity at these sites. However, mushroom fruiting bodies from neither site showed identical genotypes between the two consecutive years.

\section{Limited evidence for pseudohomothallic reproduction and inbreeding at either site}

The mean numbers of basidiospores per basidium were $2 \cdot 18$ (range 2.03-2.87 among the 22 strains) for site 1 ; and 2.34 (range 2.03-3.77 among the 28 strains) for site 2. One strain, BS261 from site 2, had a mean spore number per basidium of $3 \cdot 77$. For all other 49 strains, the majority of the basidiospores were bi-nucleate and selffertile. Our prediction therefore was that many pairs of strains reflecting pseudohomothallic reproduction or inbreeding should be found at both sites. Such strains are marked by two genetic characteristics: (i) both isolates carry the same mitochondrial haplotype; and (ii) compared to the first-year strains, the second-year strains should show identical genotype or loss of heterozygosity at certain loci. These two criteria assumed that mutations at the examined genomic regions are negligible over the 2-year span, the duration of available sample. This assumption is most likely valid. Monthly laboratory transfers of the same strain over a 2-year period revealed that transferred clones had identical genotype to the parental clone at the examined RFLP loci (J. Xu, unpublished data).
Two pairs of strains from site 2 are consistent with the predictions of pseudohomothallic reproduction in nature. The first pair consisted of strains BS258 and BS355; with strains BS355 showing loss of heterozygosity at locus $4 \mathrm{n} 6$. The second pair consisted of strains BS259 and BS370, with strain BS370 showing loss of heterozygosity at locus Pgm (Table 1). No evidence for pseudohomothallic reproduction was found at site 1 (Table 1). Aside from the multilocus genotype comparisons, the higher observed heterozygosity than those expected under random mating in three of the four samples were also inconsistent with significant pseudohomothallic reproduction of $A$. bisporus in nature.

\section{Evidence for outcrossing and recombination in all four samples}

Table 4 presents the summary of HWE and GE tests. Overall, more than half of the 18 loci in each sample had genotype frequencies not significantly different from those expected under random mating. More strikingly, the genotypic disequilibrium tests showed that a significant proportion of loci were in GE (Table 4). Results from both tests were consistent with the hypothesis that outcrossing and recombination have played significant roles in the populations of $A$. bisporus at the two sites.

The 18 nuclear loci analysed here were located on different linkage groups (LGs, see footnote to Table 1), with loci 1n148, PR5, PR2, PR6 and 1n147 located on linkage group I (LG 1), the same LG as the mating-type locus. Loci linked to mating-type locus could show high levels of heterozygosity because of the potential hitchhike effects of frequency-dependent selection on the mating-type locus. Therefore, we tested if loci on LG I have significantly elevated heterozygosity as compared to loci on other LGs. However, no statistically significant differences were observed for either observed or expected heterozygosities $(P>0 \cdot 1)$. Heterogeneity was 
Table 4. Tests of HWE and GE in four samples of $A$. bisporus from two sites in France

\begin{tabular}{|c|c|c|c|c|}
\hline \multirow[t]{2}{*}{ Allelic association indices } & \multicolumn{2}{|c|}{ Site 1, Gradignan } & \multicolumn{2}{|c|}{ Site 2, Dinard } \\
\hline & 1991 & 1992 & 1992 & 1993 \\
\hline Number of loci in HWE (out of 18 total loci) & 13 & 11 & 10 & 12 \\
\hline $\begin{array}{l}\text { Number of pairs of loci in GE (out of } 153 \text { total } \\
\text { possible pairs) }\end{array}$ & 152 & 127 & 131 & 153 \\
\hline
\end{tabular}

observed among loci from both within and between different LGs. The four samples also varied greatly in their patterns of heterozygosity.

\section{Changes of genes and gene frequencies from one year to the next}

Aside from the lack of identical genotypes between the two years at both sites, there were significant changes in genes and gene frequencies. Most notable was the number of alleles present in one year but absent in the other. For example, at site 1, 10 of the 53 nuclear alleles in the 1991 sample were absent in the 1992 sample, but the 1992 sample contained six additional alleles not found in the 1991 sample. Similar results were observed at site 2. Compared to the 1992 sample, 13 alleles were not observed but two new alleles were found in the 1993 sample. Aside from the allelic differences, a range of allele frequency changes was also observed. For example, at site 1 , allele 4 at locus $4 \mathrm{n} 6$ decreased by $40 \%$ and allele 3 increased by $48 \%$ (Table 1 ).

The genetic differences in the nuclear genome from one year to the next were paralleled by the changes in the mitochondrial haplotypes at both sites. For example, at site 1 , two additional mtDNA haplotypes (D and $\mathrm{E}$ ) were found in the 1992 sample but absent in the 1991 sample. At site 2, three mtDNA haplotypes (C, D, F) present in the 1992 sample were not found in the 1993 sample. However, one new mtDNA haplotype (I) was found in the site 2, 1993 sample (Table 1).

\section{DISCUSSION}

In this study, we examined a collection of A. bisporus natural isolates and determined their multilocus genotypes at 18 polymorphic nuclear loci and the mitochondrial genome. Though our sample sizes are relatively small, this unique collection allowed us to infer the mode of reproduction and the fine-scale spatialtemporal genetic variation of A. bisporus in its natural environment. A high level of genetic diversity was found within each of the samples. The large number of highly polymorphic loci examined here allowed unambiguous identification of genotypes.

The results here demonstrated limited evidence for vegetative clonality of A. bisporus in nature. Among the four samples, two (the site 1, 1991 and site 2, 1993) had mushroom fruiting bodies all with different multilocus genotypes. While fruiting bodies with identical genotypes were found in the other two samples, those from the site 1, 1992 sample were all clustered. The largest potential genet (consisting of strains BS264, BS265 and BS266 from the site 2, 1992 sample) was $1.3 \mathrm{~m}$ in one dimension. Interestingly, this genotype was not recovered in the second year at this site. In addition, when we examined the Cupressus leaf litter around mushroom fruiting bodies, we generally observed discoloured litter less than $50 \mathrm{~cm}$ in diameter, suggesting that A. bisporus mycelia were poorly extended in nature (Callac, 1994). Taken together, the data here suggest that vegetative clonality is limited for A. bisporus in nature.

Such an apparent lack of vegetative clonality at both sites contrasts with many other basidiomycete species (Hansen \& Hamelin, 1997). For example, a single clone of the root-rot mushroom, Armillaria gallica, can occupy several hectares in area and over 1500 years in age (Smith et al., 1992). However, basidiomycete species lacking extensive vegetative clonality are not uncommon (Hansen \& Hamelin, 1997). For example, the typical genet size of the ectomycorrhizal mushroom Hebeloma cyclindrosporum was about $50-70 \mathrm{~cm}^{2}$ (Guidot et al., 2001). In addition, most studies where large genets were found used only few and often genetically undefined markers to distinguish genets (see review by Hansen $\&$ Hamelin, 1997). If more genetically well-defined and highly polymorphic markers were used, genet sizes in some of those species could be smaller. We would also like to point out that the small genet size as discovered here for A. bisporus did not exclude the possibility that basidiospores and possibly mycelia in A. bisporus could persist for long periods of time in nature.

Interestingly, there was only limited evidence of pseudohomothallic reproduction or inbreeding in A. bisporus in nature. This result was surprising given our current understandings of the life cycle of A. bisporus and what we observe of basidiospore numbers from these isolates. Furthermore, this limited evidence for pseudohomothallic reproduction or inbreeding contrasts with the abundant evidence for outcrossing and recombination at both sites (Table 4). There are several possible explanations for the observed patterns of genetic variation. The first is that outcrossing is more common than previously thought. Even though only a small proportion (typically less than $10 \%$ ) of homokaryotic basidiospores were produced by most fruiting bodies (Kerrigan et al., 1993; Summerbell et al., 1989), if 
suitable mating partners existed, outcrossing among these self-sterile homokaryons could generate novel genotypes capable of out-competing strains from heterothallic spores (Xu et al., 1996a; Xu, 1995). Given that one mushroom fruiting body can produce about $10^{9}$ spores, if $5 \%$ of those were homokaryotic, $5 \times 10^{7}$ selfsterile spores is a big number and could provide abundant opportunity for outcrossing. In addition, some strains (e.g. BS261, see Callac et al., 1998) had predominantly four-spored basidia and a significant proportion of those spores were capable of mating with other homokaryons carrying compatible mating types.

The second possibility was that mating between homokaryons and heterokaryons could also occur to generate novel genotypes. In a laboratory investigation, genetic recombination was commonly found from pairings between homokaryons and heterokaryons (Xu et al., 1996b). The third possibility was that mating and recombination could also occur in pairings involving unrelated heterokaryons (Xu et al., 1996b). Indeed, the matings between homokaryons and homokaryons, between homokaryons and heterokaryons and between heterokaryons and heterokaryons could all contribute to the generation of novel genotypes and to the maintenance of high levels of genetic variation in these populations. Aside from the mechanisms of outcrossing, the time and frequency of outcrossing in populations of A. bisporus are also contentious issues remaining to be resolved. More extensive sampling of mushroom fruiting bodies as well as spores and mycelia is needed to address these questions. Unfortunately, fruiting is an uncommon event for A. bisporus in nature and no method is available to directly isolate and analyse spores and mycelia from its natural habitats. Putative hybrids have also been reported in a coastal Californian population of A. bisporus (Kerrigan et al., 1998).

Unlike rhizomorphic fungi where vegetative mycelium could be easily sampled and used for population genetic analysis, our sampling and analyses of A. bisporus were limited only to mushroom fruiting bodies. Though we attempted to obtain as many fruiting bodies as possible from these two sites and other sites in France between 1991 and 1994, samples from these two sites represent the best we were able to obtain for fine-scale spatialtemporal analysis. Undoubtedly, what we observed here represented only a small part of the genetic diversity within each site. Significant genetic diversity could exist in the forms of dormant spores and mycelia that escaped our sampling. If more sensitive sampling methods were developed in the future for spores and mycelia of A. bisporus, analyses of these samples might reveal a population structure very different from what we observed here for mushroom fruiting bodies. Furthermore, in combination with fruiting body samples, the analysis of mycelia samples over an extended period of time could allow us to address other potential questions regarding the length of reproductive cycle, the maintenance of vegetative mycelium in the natural environments and the roles of natural selections in populations of A. bisporus.
Our results also demonstrated that genetic drift within a population and gene flow among neighbouring populations could contribute to gene and genotype changes. However, the significant differentiation between the two sites suggests that, aside from cultivar escapes, longdistance gene flow was relatively limited (Table 3; see also Xu et al., 1997). Interestingly, the overall temporal genetic variations were similar between the two sites. For example, both sites showed abundant genotype and gene frequency changes over the 2-year period. However, there were some differences. First, the overall observed heterozygosity was higher at site 2 than at site 1 (Table 2). Second, while no evidence of pseudohomothallic reproduction or inbreeding was observed at site 1 , two pairs of strains at site 2 were consistent with pseudohomothallic reproduction and inbreeding. The frequent disturbance experienced at site 1 could have contributed to these differences.

Observed heterozygosities were higher than expected under random mating in three of the four samples (Table 2). Several factors could have contributed to the excess heterozygotes, including (i) linkage to the matingtype locus, (ii) close proximity to centromeres and (iii) selection for heterozygotes. In A. bisporus, the matingtype locus was mapped to LG 1 (Xu et al., 1993). Five of the 18 loci analysed here are on LG 1 (see footnote to Table 1). Because mating-type alleles are under frequency-dependent selection, loci linked to mating type could have a significantly higher observed heterozygosity than expected because of hitchhiking effects. However, our data showed little consistent pattern of heterogeneities among loci from within and between different LGs (Table 1). Another factor that could influence heterozygosity is distances between loci and centromeres. The closer loci are to their respective centromeres, the less likely crossing over will occur and therefore, heterozygosities are more likely to be preserved. However, the positions of centromeres are not known for any of the LGs in A. bisporus. The third factor is the potential selection for heterozygotes or against homozygotes. A previous investigation showed that outcrossing and heterozygosity were positively correlated to fitness in A. bisporus under laboratory conditions (Xu, 1995). However, there is currently no direct evidence of natural selection for excess heterozygosity in natural populations of A. bisporus.

The observation of significant evidence for mating and recombination in natural populations of A. bisporus calls into question the origin and maintenance of the predominant pseudohomothallic life cycle in this species. One possible explanation is that heterokaryotic basidiospores were abundantly produced and germinated but the establishment of these heterokaryotic mycelia was soon followed by either homokaryon-heterokaryon or heterokaryon-heterokaryon matings. Heterokaryotic spores generally have higher germination rates than homokaryotic spores and heterokaryotic mycelia have greater vegetative growth rates than homokaryotic mycelia. Therefore, advantages afforded by heterokaryotic spores could contribute to 
the origin and maintenance of the pseudohomothallic reproduction in A. bisporus.

In conclusion, we found high levels of genetic diversity in all four samples of A. bisporus from the two French sites. This high genetic diversity along with the large number of polymorphic loci analysed enabled us to address several questions concerning the modes of reproduction and the fine-scale spatial-temporal patterns of genetic variation of A. bisporus in nature. Our analyses indicated that natural vegetative clones were small in A. bisporus and that strict pseudohomothallic reproduction was not common in nature. Instead, the data here indicated that outcrossing and recombination have played significant roles in the genetic structure in natural populations of A. bisporus.

\section{ACKNOWLEDGEMENTS}

This research was supported by the Canadian Mushroom Growers' Association (J. Xu), the French Centre Technique du Champignon (C. Desmerger), the Bureau des Ressources Génétiques (BRG) in France (P. Callac) and the Natural Science and Engineering Research Council (NSERC) of Canada (J. Xu). We thank Jim Anderson, Paul Horgen, Rick Kerrigan and Heather Yoell for their support and advice at various stages of this study. The technical assistance of Sandra Hainaut and Lucette Pirobe was greatly appreciated.

\section{REFERENCES}

Allen, J. J., Moore, D. \& Elliott, T. J. (1992). Persistent meiotic arrest in basidia of Agaricus bisporus. Mycol Res 96, 125-127.

Callac, P. (1994). Prospections pour la recherche d'Agaricus bisporus en France: contexte historique et scientifique, premiers résultats. Bull Soc Mycol Fr 110, 145-165.

Callac, P. (1995). Breeding of edible fungi with emphasis on the variability among French genetic resources of Agaricus bisporus. Can J Bot 73 (Suppl. 1), S980-S986.

Callac, P., Desmerger, C., Kerrigan, R. W. \& Imbernon, M. (1997). Conservation of genetic linkage with map expansion in distantly related crosses of Agaricus bisporus. FEMS Microbiol 146, 235-240.

Callac, P., Hocquart, S., Imbernon, M., Desmerger, C. \& Olivier, J. M. (1998). Bsn-t allele from French field strains of Agaricus bisporus. Appl Environ Microbiol 64, 2105-2110.

Callac, P., Imbernon, M., Guinberteau, J., Pirobe, L., Granit, S. \& Olivier, J.-M. (2000). Discovery of a wild Mediterranean population of Agaricus bisporus, and its usefulness for breeding work. Mushroom Sci XV, 245-252.

Chang, S. T. (1993). Mushroom biology : the impact on mushroom production and mushroom products. In Mushroom Biology and Mushroom Products, pp. 3-20. Edited by S. T. Chang, J. A. Buswell \& S. W. Chiu. Hong Kong: The Chinese University of Hong Kong Press.

Guidot, A., Debaud, J. C. \& Marmeisse, R. (2001). Between genet diversity and spatial distribution of above- and below-ground populations of the ectomycorrhizal fungus Hebeloma cylindrosporum. Mol Ecol 10, 1121-1131.

Guinberteau, J., Callac, P. \& Boisselet, P. (1998). Inventaire des communautés fongiques liées au Cupressus macrocarpa en zone littorale atlantique et données récentes sur les populations sauvages d'Agaricus bisporus. Bull Soc Mycol Fr 114, 19-38.

Hansen, E. M. \& Hamelin, R. C. (1997). Population structure of
Basidiomycetes. In Structure and Dynamics of Fungal Populations, pp. 251-281. Edited by J. Worral. Dordrecht: Kluwer.

Hintz, W. E. A., Anderson, J. B. \& Horgen, P. A. (1988). Physical mapping of the mitochondrial genome of the cultivated mushroom Agaricus brunnescens ( $=$ A. bisporus). Curr Genet 14, 43-49.

Imbernon, M., Callac, P., Granit, S. \& Pirobe, L. (1995). Allelic polymorphism at the mating type locus in Agaricus bisporus var. burnettii, and confirmation of the dominance of its tetrasporic trait. Mushroom Sci XIV, 11-19.

Jin, T. \& Horgen, P. A. (1993). Further characterization of a large inverted repeat in Agaricus bisporus ( $=$ A. brunnescens) and related species. Curr Genet 23, 228-233.

Kerrigan, R. W. (1995). Global genetic resources for Agaricus breeding and cultivation. Can J Bot 73 (Suppl. 1), S973-S979.

Kerrigan, R. W., Royer, J. C., Baller, L. M., Kohli, Y., Horgen, P. A. \& Anderson, J. B. (1993). Meiotic behavior and linkage relationships in the secondarily homothallic fungus Agaricus bisporus. Genetics 133, 225-236.

Kerrigan, R. W., Billette, C., Callac, P. \& Velcko, T. J., Jr (1996). A summary of allelic diversity and geographical distribution at six allozyme loci of Agaricus bisporus. In Mushroom Biology and Mushroom Products, pp. 25-35. Edited by D. Royse. Proceedings of the Second International Conference, University Park, PA, USA.

Kerrigan, R. W., Carvalho, D. B., Horgen, P. A. \& Anderson, J. B. (1998). The indigenous coastal Californian population of the mushroom Agaricus bisporus, a cultivated species, may be at risk of extinction. Mol Ecol 7, 35-45.

Lange, M. (1952). Species concept in the genus Coprinus. Dansk Bot Ark 14, 1-140.

Mouyna, I. \& Brygoo, Y. (1993). Les champignons et la PCR. In $\mathrm{La}$ PCR: un Procede de Replication in vitro, pp. 335-341. Edited by D. Larzul. Paris: Technique et Documentation, Lavoisier.

Raper, C. A., Raper, J. R. \& Miller, R. E. (1972). Genetic analysis of the life cycle of Agaricus bisporus. Mycologia 64, 1088-1117.

Reynolds, J., Weir, B. S. \& Cockerham, C. C. (1983). Estimating the coancestry coefficient: basis for a short-term genetic distance. Genetics 105, 767-779.

Royse, D. J. \& May, B. (1982). Genetic relatedness and its application in selective breeding of Agaricus brunnescens. Mycologia 74, 569-575.

Smith, M. L., Bruhn, J. N. \& Anderson, J. B. (1992). The fungus Armillaria bulbosa is among the largest and oldest living organisms. Nature 356, 428-431.

Summerbell, R. C., Castle, A. J., Horgen, P. A. \& Anderson, J. B. (1989). Inheritance of restriction fragment length polymorphisms in Agaricus brunnescens. Genetics 123, 293-300.

Weir, B. S. \& Cockerham, C. C. (1984). Estimating F-statistics for the analysis of population structure. Evolution 38, 1358-1370.

Xu, J. (1995). Analysis of inbreeding depression in Agaricus bisporus. Genetics, 141, 137-145.

Xu, J., Kerrigan, R. W., Horgen, P. A. \& Anderson, J. B. (1993). Localization of the mating type gene in Agaricus bisporus. Appl Environ Microbiol 59, 3044-3049.

Xu, J., Horgen, P. A. \& Anderson, J. B. (1996a). Variation of mating interactions in Agaricus bisporus. Cultivated Mushroom Res Newsl 3, 23-30.

Xu, J., Horgen, P. A. \& Anderson, J. B. (1996b). Somatic recombination in the cultivated mushroom Agaricus bisporus. Mycol Res 100, 188-192. 
Xu, J., Kerrigan, R. W., Callac, P., Horgen, P. A. \& Anderson, J. B. (1997). The genetic structure of natural populations of Agaricus bisporus, the commercial mushroom. J Hered 88, 482-494.

Xu, J., Kerrigan, R. W., Sonnenberg, A. S., Callac, P., Horgen, P. A. \& Anderson, J. B. (1998). Mitochondrial DNA variation in natural populations of the mushroom Agaricus bisporus. Mol Ecol 7, 19-33.
Zaykin, D., Zhivotovsky, L. \& Weir, B. S. (1995). Exact tests for association between alleles at arbitrary numbers of loci. Genetica 96, 169-178.

Received 17 October 2001; revised 19 December 2001; accepted 7 January 2002. 